\title{
Frontiers in Bladder Cancer Genomic Research
}

\author{
Yi $\mathrm{Li}^{1 \dagger}{ }^{1 \dagger}$, Lihui Sun ${ }^{2 \dagger}$, Xiangyang Guo ${ }^{1}, \mathrm{Na} \mathrm{Mo}{ }^{3}$, Jinku Zhang ${ }^{4,5 *}$ and Chong $\mathrm{Li}^{2,5,6,7 *}$ \\ ${ }^{1}$ Department of Anesthesiology, Peking University Third Hospital, Beijing, China, ${ }^{2}$ Core Facility for Protein Research, Institute \\ of Biophysics, Chinese Academy of Sciences, Beijing, China, ${ }^{3}$ Department of Pathology, Beijing Obstetrics and Gynecology \\ Hospital, Capital Medical University, Beijing, China, ${ }^{4}$ Department of Pathology, First Central Hospital of Baoding, \\ Baoding, China, ${ }^{5}$ Key Laboratory of Molecular Pathology and Early Diagnosis of Tumor in Hebei Province, First Central \\ Hospital of Baoding, Baoding, China, ${ }^{6}$ Department of Immunology, Beijing Jianlan Institute of Medicine, Beijing, China, \\ 7 Department of Immunology, Beijing Zhongke Jianlan Biotechnology Co., Ltd., Beijing, China
}

\section{OPEN ACCESS}

Edited by:

Meng Zhang,

First Affiliated Hospital of Anhu Medical University, China

Reviewed by:

Guru Sonpavde,

Dana-Farber Cancer Institute, United States

Takeshi Yuasa,

Japanese Foundation For Cancer

Research, Japan

*Correspondence:

Jinku Zhang

843561234@qq.com

Chong Li

lichong@moon.ibp.ac.cn

${ }^{\dagger}$ These authors have contributed equally to this work

Specialty section:

This article was submitted to Genitourinary Oncology,

a section of the journal

Frontiers in Oncology

Received: 22 February 2021

Accepted: 28 April 2021

Published: 20 May 2021

Citation:

Li Y, Sun L, Guo X, Mo N,

Zhang $J$ and Li C (2021)

Frontiers in Bladder Cancer

Genomic Research.

Front. Oncol. 11:670729.

doi: 10.3389/fonc.2021.670729
Most of the etiology studies of bladder cancer focus on genetic changes, mainly including mutation and activation of oncogenes, mutation and inactivation of tumor suppressor genes, and rearrangement or heterozygous deletion of chromosomes. Moreover, bladder cancer is highly heterogeneous mainly due to abnormal changes in the genome and proteome of tumor cells. Surgery is the main treatment for bladder cancer, but because the recurrence rate is high after surgery and most of the muscle-invasive bladder cancer acquires distant metastasis. Therefore, there is a need to combine with chemotherapy to consolidate the treatment effect. However, there are differences in chemosensitivity among patients. In this article, we review the up-to-date genomic researches on bladder cancer occurrence, development, metastasis, and chemosensitivity in patients, in order to provide some theoretical support for the diagnosis and treatment strategy for bladder cancer.

Keywords: bladder cancer genomics, heterogeneity, epigenetics, oncogene sequencing method, precision treatment

\section{INTRODUCTION}

Bladder cancer is the ninth most common malignant disease worldwide, with 549,393 new cases reported in 2018, and it ranks fourteenth in cancer mortality worldwide. Moreover, its incidence and mortality in males increased to the 6th and 9th place among cancers, respectively. Although men are more likely to develop bladder cancer, women often present with more advanced disease and have unfavorable prognosis. This disease can present as non-muscle-invasive bladder cancer (NMIBC), muscle-invasive bladder cancer (MIBC) and a metastatic form of the disease. The overall survival declines dramatically as the cancer progresses, especially when urothelial cells transition from noninvasive to invasive $(1,2)$. Each stage of the disease has different molecular drivers, and epigenetic dysregulation also plays an important role in the pathogenesis of bladder cancer (3). Furthermore, heterogeneity is a characteristic feature of bladder cancer, which exhibits a wide spectrum of clinical and pathologic features (4). In addition, chemotherapy is an important treatment method for bladder cancer, but chemotherapy has failed in a large proportion of patients with bladder cancer because of the gradual chemoresistance, which leads to the relapse and progression of tumors (5).

Recent advances in the next-generation sequencing technologies have significantly improved our understanding of the genomic landscape and the molecular underpinnings of bladder cancer (6). 
This review will summarize the molecular mechanism of bladder cancer occurrence, development and metastasis, as well as the sensitivity towards chemotherapy in patients, in order to pave the way for diagnosis, monitoring, prognosis, and personalized care for bladder cancer. Meanwhile, the novel oncogene sequencing method and strategy will also be discussed.

\section{BLADDER CANCER GENOMICS}

Carcinogenesis of cells is a very complicated process (7). At the genomic level, its cancerous mechanisms are mainly involved the following aspects: oncogene activation and overexpression; tumor suppressor gene mutations and deletions; loss of gene repair function; tandem duplication of nucleotide abnormalities in the genome, Microsatellite instability; dysfunction of signaling pathways; cell telomerase overexpression. In 2005, the United States launched the Oncology Genome Research Program (4), and hopes to find out all the oncogene in lung cancer, brain cancer, and ovarian cancer in the next 13 years in order to diagnose and treat these "terminally diseases". However, each tumor has its own unique genetic blueprint, even the cause of same tumor type is different between individuals. Scientists hope to use the "Tumor Genome Project" to establish global collaboration to unravel the secrets of all cancers and build a shared database. The successful implementation of this program is expected to truly benefit human health.

Bladder cancer genomics is a discipline based on the study of the bladder cancer genome to reveal the mechanism of bladder cancer development. Bladder cancer genomics can be divided into two aspects (8): to identify new mutation sites, genes and corresponding molecular signaling pathways related to bladder cancer, and to analyze the causes of tumorigenesis and development from the gene mutation spectrum.

\section{Bladder Cancer Genomics}

Gene abnormalities can lead to cell cycle disorders, uncontrolled proliferation, and thus tumors. The genomic defects of bladder cancer are complicated, ranging from single DNA mutations to gene polymorphisms to whole or partial deletions of chromosomes.

The study has found that all human malignancies have somatic gene mutations (9). Activation of oncogenes and suppression of tumor suppressor genes are very common. Abnormal activation of oncogenes such as HER2 can facilitate tumor metastasis. Inactivation of p53 and p16 and other tumor suppressor genes involved in cell cycle regulation can inhibit apoptosis of tumor cells. Common mutations in bladder cancer include TP53, PIK3CA, TSC1, FGFR3, HRAS, and HER2. Moreover, common abnormal expression genes include $E G F R$, Ki67, PD-L1, ERCC1, and BRCA1. Shariat et al. found that the proportion of $p 53$ and $p 16$ abnormally expressed in bladder cancer patients were $56 \%$ and $54 \%$, respectively. It was highly correlated with muscle-invasive bladder cancer which often indicates a poor prognosis (5). Soria et al. studied the expression of HER2 in 354 bladder cancer patients and found that HER2 is highly expressed in more than $32 \%$ of patients. Higher expression of HER2 indicates more aggressive tumor invasiveness (10). Hayashi et al. inhibited highly expressed HER2 in bladder cancer cell lines and animal models with HER2 inhibitor T-DM1 and found that T-DM1 has a more prominent inhibitory effect on bladder cancer cell growth than the trastuzumab. In addition, platinum-resistant bladder cancer cells with have higher levels of HER2 expression. Interestingly, this group of cells is more sensitive to T-DM1, and cells treated with T-DM1 are more prone to apoptosis (11). The above studies indicate that the oncogene HER2 plays an important role in the pathogenesis of bladder cancer. Inhibition of HER2 expression and induction of apoptosis can significantly suppress the bladder cancer cell growth. HER2 mutations are also present in bladder cancer. Tschui et al. studied both exon 19 and exon 20 of HER2 gene in bladder cancer patients and found that mutations occur in exon 19 (12).

The cancer genome atlas (TCGA) analyzed DNA data from 131 patients with muscle-invasive bladder cancer and identified 32 mutant genes with high frequency. These mutations are mainly occurred in the cell cycle, chromatin regulation and kinase signaling pathways (13). Another comprehensive analysis of the full TCGA cohort of 412 MIBC cases was conducted in 2017. 58 significantly mutated genes (SMGs) were identified in the expanded cohort, in which 34 mutant genes were not identified in previous analysis and 7 mutant genes were identified in more than $10 \%$ of samples: KMT2A (11\%), SPTAN1 (12\%), ERBB2 (12\%), CREBBP (12\%), FAT1 (12\%), ATM (14\%) and KMT2C (18\%). Moreover, 158 genes that were epigenetically silenced were identified by analyzing DNA methylation and gene expression, for example, SPATC1L (silenced in 19\%), nicotinate phosphoribosyltransferase (NAPRT) (13\%), Poly(ADP-ribose) polymerase PARP6 (26\%) and latexin $(L X N)(27 \%)$. However, the DNA hypermethylation in the promoter of other tumor suppressor genes were not found, such as RB1, NF2, NF1, TSC2, TSC1, PTEN, and TP53 (14).

In addition to single DNA abnormality, bladder cancer genomic research also focuses on polymorphism sites. There are multiple gene polymorphisms in bladder cancer such as ERCC1, XRCC1, GSTP1, CDA, GSTM1, and GSTT. Different gene polymorphisms could predict chemotherapy sensitivity in bladder cancer. $\mathrm{Xu}$ et al. studied the efficacy of 41 patients at stage IV of muscle-invasive bladder cancer with different ERCC1 genotypes after 2 to 6 cycles of platinum-based chemotherapy. The results suggest there is a discrepancy of short-term response to chemotherapy and median overall survival in patients with different ERCC1 genotype. Compared with $\mathrm{C} / \mathrm{T}$ and $\mathrm{T} / \mathrm{T}$ genotypes, patients with $\mathrm{C} / \mathrm{C}$ genotype had a better short-term response to chemotherapy and median overall survival (15).

Studies have shown that there are changes in the PI3K, MAPK, Hedgehog, and Wnt pathways in the bladder cancer genome (16-18). Among them, the PI3K pathway is the most studied signaling pathway in bladder cancer. ERBB receptor family is the upstream promoter of the PI3K pathway. For example, ERBB1 (EGFR) can activate the PI3K pathway by activating RAS. Overexpression of EGFR, ERBB2 or ERBB3 is 
associated with tumor grade, stage, and prognosis in bladder cancer. Moreover, mutations in ERBB2 or ERBB3 have been found in some muscle-invasive bladder cancers (19). PIK3CA encodes the catalytic subunit $\mathrm{p} 110 \alpha$ of PI3K, and its mutation occurs in $25 \%$ of non-muscle invasive bladder cancer (20). The above studies suggest that the mechanism of bladder cancer development and progression may be related to the PI3K signaling pathway.

\section{Genetics of Bladder Cancer Cell}

Chromosomes are aggregates of genetic material, and chromosomal abnormalities are an important part of the genetic defects of bladder cancer. Based on genetic techniques such as genomic hybridization and loss of heterozygosity, it is found that bladder cancer has complex chromosome number and structural variation. Bartoletti et al. have shown that the partial or complete loss of genetic material at chromosome 9 can lead to the loss of tumor suppressor genes such as $p 16$ which often indicates the recurrence of low-grade bladder cancer. Abnormal numbers of chromosomes 3, 7, 13, and 17 have also occurred in bladder cancer (21). Among them, chromosome 3 polyploid and chromosome 7 aneuploidy may be related to the progression and malignancy of bladder cancer, and the aneuploidy of chromosome 17 often implies a high recurrence rate of bladder cancer $(4,22)$.

\section{HETEROGENEITY OF BLADDER CANCER GENOME}

Tumor heterogeneity describes differences of genotype and phenotype between the same tumor type in different patients, and different sites in an individual, and even between the cancer cells within a tumor. For different individuals, tumor heterogeneity mainly depicts as differences in clinical characteristics such as pathological type, the degree of malignancy, invasion, and metastasis as well as gene mutation, and abnormal expression of proteins. For the same individual, tumor heterogeneity mainly exhibits as differences in gene expression profile and mutation at different sites of the same individual or between the cells of the same tumor, even between many subtypes of tumor cells in the tumor. Phenotypic and genomic (genetic) heterogeneity are two manifestations of tumor heterogeneity. Phenotypic heterogeneity is also the result of genomic heterogeneity to some extent (23). Therefore, the key to the study of tumor heterogeneity lies in genomic heterogeneity research.

According to the biological behavior of bladder cancer, it can be divided into muscle-invasive bladder cancer (MIBC) and nonmuscle invasive bladder cancer (NMIBC). Studies have shown that there is a mutation of Ha-ras/FGFR3 gene in NMIBC, and activation of the mouse $\mathrm{Ha}$-ras gene can induce NMIBC, which is rare in MIBC. Inactivation of the $p 53 / R b / P T E N$ gene is more likely happen in MIBC. Activation of the uroplakin II-specific urothelial-specific promoter in transgenic mice demonstrated the expression of SV40T antigen in the urothelium which can inactivate $p 53$ and $p R b$, thereby induces the invasion of tumor and metastasis of bladder cancer. Inactivation of $p 53 / R b / P T E N$ is less common in non-muscle invasive bladder cancer $(24,25)$. These findings suggest that the genomic heterogeneity (mutation and expression profile) between MIBC and NMIBC is likely to be significantly different in the biological behavior of the two bladder cancers.

Tumor heterogeneity can also exhibit as heterogeneity at the stage of tumor development. The infinite proliferation of cells is one of the main features of malignant tumors, and this characteristic requires de novo synthesis of telomeres by telomerase to prolong the loss of telomere ends in each round of DNA replication. $\mathrm{Wu}$ et al. performed whole genome and transcriptome sequencings of 97 bladder cancer patients and found that the telomere reverse transcriptase gene TERT is highly expressed in invasive and advanced bladder cancer patients as compared to the early and non-invasive bladder cancer patients. This group of patients has a higher mutation rate of the TERT promoter (26). This study showed a significant difference in the TERT promoter expression level and the mutation rate between early non-invasive and advanced invasive cancerous cells in bladder cancer.

Bladder cancer tumors have different biological characteristics of the tumor cells subsets, and there are certain differences in gene expression profiles among different cell subpopulations, which is well-described by the heterogeneity within the tumor. The most representative of intratumoral heterogeneity is the presence of a subset of tumor cells with different molecular markers in the bladder cancer. For instance, bladder cancer cells can be divided into common bladder cancer cells and bladder cancer stem cells, with a significant difference in genetic mutations at the latter. $\mathrm{Li}$ et al. extracted the single cell genomes of bladder cancer stem cells (BCSCs) and nonbladder cancer stem cells (non-BCSCs) for PCR amplification, and analyzed data from 20 cells. The single cell mutation rate of the TERT gene promoter C228T was found to be $50 \%$ higher in BCSCs and lower in non-BCSCs (27). There are two main hypotheses of the formation mechanism of malignant tumors heterogeneity in bladder cancer: clonal evolution hypothesis and cancer stem cell hypothesis $(28,29)$. The clonal evolution hypothesis was proposed by Nowell in 1976 which hypothesize that tumor cells originate from the first generation of single mutant cells. Thus, all tumor cell genomes are identical to the genome of the single cell, but complex factors such as mutations and environmental influences are involved in the proliferation and evolution process. Therefore, the subsequent formation of tumor cells is gradually varied in term of genotypes and phenotypes and eventually result in the tumor heterogeneity. Cancer stem cells are a research hot topic in molecular biology of tumor in recent years. The cancer stem cell hypothesis states that tumor cells are derived from cancer stem cells. Due to the multi-directional differentiation potential of tumor stem cells, various tumor cells proliferate and differentiate to produce subpopulation with different phenotypic functions and thus result in tumor heterogeneity. Kreso et al. found that the mutated genes of tumor cells in the same colorectal cancer case remained unchanged after continuous multiple implantations, 
but there were significant differences in phenotypes such as proliferative capacity and drug resistance (30). However, this hypothesis does not explain why there are differences in the genome of tumor cells of the same tumor type between different individuals.

Tumor heterogeneity is the result of genetic and environmental interactions. Although the current hypothesis does not completely reveal the cause of tumor heterogeneity, it offers research direction for tumor heterogeneity to some extent.

\section{EPIGENETIC AND BLADDER CANCER GENOME}

\section{Telomerase Reverse Transcriptase}

Telomerase is a ribonucleoprotein that is essential for the replication of most eukaryotic chromosome ends. In cancer cells, telomerase can be activated. In contrast, telomerase is epigenetically silenced and inactivated in normal cells (31). However, mutations of important genes can reverse telomerase silencings, such as tumor suppressor genes or mutations in the tumor suppressor pathway signaling molecules that may affect telomerase activity in human tumors (32). Telomerase consists of two subunits: telomerase RNA component (TERC) and telomerase reverse transcriptase tert (TERT) catalytic subunit. The TERT gene encodes a telomerase reverse transcriptase catalytic subunit and assembles into a ribonucleoprotein protease complex to maintain telomere length which plays an important role in the maintenance of tumor genome stability (33). In recent years, TERT promoter mutations have been found in malignant tumors such as melanoma (34) and glioma (35). Studies have shown that the TERT promoter mutation is highly correlated with the prognosis of transitional cell carcinoma and confirmed that the $-124 \mathrm{C}>\mathrm{T}$ mutation (corresponding to C228T) is associated with high expression of TERT and telomerase activity (36).

$\mathrm{Wu}$ et al. found a coexistence relationship between the TERT promoter mutation point and the TC53/RB1 inert somatic mutation point. By determining the chromosomal instability index, it was found that the tumor chromosomal instability index of the TERT promoter mutation was significantly higher than that of the tumor without the TERT promoter mutation (26). TP53 is involved in the regulation of telomerase activity, maintenance of gene integrity, inert mutations, and its deletion is associated with chromosomal instability. These studies suggest that the coexistence of the two may have a synergistic effect on the chromosomal instability of bladder cancer (37).

Studies have found that the C228T mutation of the TERT promoter often occurs in BCSCs (27). Compared with nonBCSCs, C228T has a significantly higher mutation rate in BCSCs which is consistent with the phenomenon of high C228T expression in stem cells compared to normal somatic cells. Importantly, the TERT promoter C228T mutation can convert normal bladder stem cells (NBSCs) into tumor-initiating cells. This mutation is located at the promoter region and can lead to tumors formation which can be considered as tumor promoters.
It can be seen that the high mutation rate of TERT promoter in BCSCs is a new feature of bladder cancer, but the transformation of NBSCs mutation into BCSCs is complex and further research is needed.

Telomerase activity is observed in almost all human tumor types, thus monitoring the tumor's telomerase activity will greatly contribute to the diagnosis and screening of bladder cancer. $\mathrm{Wu}$ et al. screened for the presence of telomerase reverse transcriptase gene promoter somatic cell mutations by Sanger sequencing in 302 patients with different urological tumors. The result showed that $43 \%$ of genitourinary tumors had TERT promoter somatic mutations. This has determined high-frequency mutation hotspot of the telomerase reverse transcriptase gene promoter in clinical urogenital (26). In different types of urological organ tumors, the amplitude of somatic mutation of the TERT promoter was larger (0-63.7\%). The urinary tract cancer has the highest mutation frequency among all, while the prostate cancer showed no mutation.

\section{Chromatin Remodeling}

Chromatin remodeling is an important regulatory mechanism of epigenetics, which is characterized by changes in the nucleosome structure and its relative position to the DNA sequence, and alteration of the accessibility of gene promoter region sequences to further regulate gene expression. Chromatin remodeling works primarily through two pathways: ATP-dependent chromatin remodeling complex, the SWl/SNF complex; histone-modifying enzymes including histone methylation, acetylation, phosphorylation, and ubiquitination (38). Recent studies have found that chromatin remodeling related genes have highfrequency mutations in a variety of tumors including bladder cancer, kidney cancer, gastric cancer, ovarian clear cell carcinoma, breast cancer and glioblastoma (38). Mutations in chromatin remodeling-related genes can cause epigenetic changes in local histone modifications and chromatin conformation in cancer cells, leading to dysregulation of downstream signaling genes, changes in biological behavior such as cell proliferation and apoptosis, leading to tumor development (39).

Gui et al. performed full exon sequencing on 9 patients with transitional cell carcinoma and has found 8 frequently mutated chromatin remodeling genes in 59\% of transitional cell bladder cancer: UTX, MLL-MLL3, CREBBP-EP300, NCOR1, ARID1A and CHD6 (40). UTX has a number of significantly enriched mutations including 11 nonsense mutations, 4 frameshift mutations, and 1 splice site change. One of these mutations was predicted to be able to truncate the JmjC domain, which is critical for the demethylase activity of the protein product. ARID1A has the same mutation pattern as UTX. In addition, mutations in the above genes have also been found in other malignant tumors such as ovarian cancer and renal cancer (41, 42). The study found that STAG2 is located on the $\mathrm{X}$ chromosome, encoding sister chromatid cohesion and segregation (SCCS) adhesion complex components, regulating the separation of sister chromosomes during cell division, and inactivation of STAG2 can cause weakening of chromosome binding and thus aneuploidy (43). Via gene sequencing and rigorous bioinformatics analysis, Guo et al. have found that 
STAG2, ESPL1 and NIPBL genes with frequent mutations in bladder cancer are involved in the SCCS process (44). The study further revealed that patients with mutant STAG2 had a higher frequency of chromosomal aneuploidy by detecting copy number changes in the chromosome arm, and significantly worse in the prognosis of STAG2 gene somatic cell mutation in bladder cancer patients compared with STAG2 bladder cancer patients without the mutation. Collectively, in SCCS, other types of tumors only report rare or low-frequency mutations in oncogenes. Bladder cancer is a tumor type that is first known to have a high-frequency genetic damage gene in the SCCS process in which the genetic mutation rate is approximately $32 \%$. The genetic changes affect the SCCS process which may involve the development of bladder cancer. Despite the detailed mechanism of STAG2 leading to the pathogenesis of transitional cell carcinoma is still not known, the highfrequency recurrent mutant gene is indeed a new pathway associated with transitional cell carcinoma in the SCCS process of transitional cell bladder cancer (45).

\section{ONCOGENE SEQUENCING METHOD AND STRATEGY}

The traditional sequencing method is Sanger sequencing. However, this method has the disadvantage of low sensitivity and it is difficult to obtain all genomic information, and high cost but low output which limits the application of Sanger sequencing in large-scale sequencing. High-throughput sequencing, the second-generation sequencing technology (also known as nextgeneration sequencing, deep sequencing) can simultaneously sequence a large number of genes, making it possible to accurately detect tumor and transcriptome abnormalities in tumor patients (46-48). This method can detect tumor genomic abnormalities including nucleotide substitution, insertion and deletion, copy number alteration and chromosome recombination, and improve the detection efficiency and resolution of the genome. As a next-generation sequencing technology, high-throughput sequencing has unparalleled advantages over the traditional sequencing methods. First, high-throughput sequencing can perform largescale parallel sequencing of a large number of genes, resulting in a remarkable increase in gene detection efficiency. Second, highthroughput sequencing detects the number of occurrences of a certain gene in a sample can reflect the expression level of the gene to some extent. Finally, high-throughput sequencing is more economical than traditional large-scale gene sequencing.

\section{Whole Genome Sequencing}

Whole genome sequencing is the sequencing of the entire genome of tumor tissue with the DNA sequence of the germ cells from the same patient (tumor cell mutation is not present in the germ cells) as a control can effectively identify the changes in all regions including nucleotide replacement, structural rearrangement, and copy number changes (49). Therefore, whole genome sequencing is the most comprehensive depiction of the tumor genome, suitable for the study of tumor genomewide association. However, whole genome sequencing requires the detection of a large number of sequences is redundant for studies that do not require the whole genome. In this case, shotgun sequencing based on the Sanger sequencing principle is more suitable for identifying somatic cell rearrangements and copy number changes in the genome.

Sanger sequencing is the gold standard for current gene sequencing. $\mathrm{Wu}$ et al. performed Sanger sequencing to sequence 302 different urinary tumor patients and found a high-frequency mutation hotspot of TERT promoter in urinary tumor patients wherein TERT promoter somatic mutation in $55.6 \%$ of bladder cancer (26). Guo et al. performed genome-wide sequencing of 99 tumors and corresponding peripheral blood samples from patients with transitional cell carcinoma by Sanger sequencing. SCCSrelated gene mutations such as STAG2 and ESPL1 were confirmed from 11240 candidate somatic mutations. Moreover, mutant genes such as FGFR3, TP53, PIK3CA, and RB1 are ubiquitous in malignant tumors (44). Second-generation sequencing invention has received extensive attention at the beginning compared to Sanger sequencing. Andrea et al. recruited 50 patients with muscle-invasive bladder cancer and performed whole-exome sequencing on germline and pretreatment tumor DNA. Then these patients were treated with neoadjuvant cisplatin-based chemotherapy and underwent surgery to evaluate the pathologic response. They identified ERCC2, one nucleotide excision repair gene, was only significantly mutated in responders compared with nonresponders (50). David et al. investigated the correlation of ERCC2 with pathologic response to neoadjuvant cisplatin-based chemotherapy in an independent validation patient cohort, and they confirmed that ERCC2 was indeed associated with response to chemotherapy (51). Desai et al. used the second-generation sequencing technology to map the mutations of primary and recurrent transitional cell bladder cancer. By comparing the differential expression of tumor-associated DNA damage response genes, they also found that especially ERCC2 mutations indicate a better prognosis of chemoradiotherapy for transitional cell bladder cancer and a lower rate of recurrence and metastasis within 2 years (52).

\section{Exon Sequencing}

Exon sequencing is a method of capturing whole genome exon DNA by using targeted sequence capture technology to enrich the construction of DNA library before performing highthroughput sequencing. It is characterized by sequencing of the open reading frame without sequencing the introns. Since exons account for only $2 \%$ of the entire genome, high-throughput sequencing of the targeted exon greatly reduces the amount of sequencing and achieves higher efficiency. Due to its deep sequencing of the coding region, it can be used to screen for mutations that Sanger sequencing could not. In addition, it is suitable for the study of single nucleotide polymorphism (SNP) and insertion-deletion (InDel) of the target gene. Longo et al. used targeted exon sequencing to detect 50 cases of patients with pTis-pT4b bladder cancer. Through the analysis of staged somatic mutations in patients with such high-risk bladder cancer, the mutated genes and mutation profiles were 
determined. In terms of single mutations, it was found that epigenetic and cell cycle-regulated genes have significantly higher mutation rates. $\mathrm{PI} 3 \mathrm{~K} / \mathrm{mTOR}$ and cell cycle/DNA repair showed the highest mutation rate among the other assessed pathways. Moreover, RB1 and TP53 mutations were found to frequently coexist with NF1 and PIK3CA mutations (53).

However, exon sequencing also has certain limitations. It is unable to sequence non-coding genes and thus fail to conduct a comprehensive study of intron-regulated genes associated with the target gene, and also impossible to identify copies.

\section{Single Cell Sequencing}

In the past, genome sequencing was the extraction of DNA from many cells or a piece of tissue. It is inevitable that sequencing results are obtained from many cell genomes. However, tumors are heterogeneous and the genomic information of different subtypes is more or less different. Investigation of the total DNA information extracted from different cell populations will inevitably cause researchers to ignore the differences between cells to varying degrees and further bias the study of the target cell genome $(54,55)$. Single cell sequencing is the sequencing of genomes from a single cell. The process involves the isolation of single cells and extraction of DNA followed by single-cell genomic amplification and sequencing analysis. This will prevent the influence of other cell genomes on the sequencing results of the target cells and thus accurately measure the copy number of a single nuclear gene (56).

Bladder cancer is a solid tumor with significant heterogeneity, and the process of research needs to be careful to prevent bias resulted by other cells. In order to study TERT promoter mutations in the bladder cancer, $\mathrm{Li}$ et al. had isolated the single cells of BCSCs, non-BCSCs, NBBCs, and non-NBBCs and extracted their genomes for single-cell gene amplification and sequencing. The high-frequency mutation sites of C228T were found in the TERT promoters of BCSCs and non-BCSCs, and the effect of C228T mutation on bladder cancer and BCSCs was investigated (27). In order to study the genetic basis and origin of bladder cancer stem cells, Yang et al. had performed single-cell sequencing on 59 single cells including bladder cancer stem cells (BCSCs), bladder cancer non-stem cells (BCNSCs), bladder epithelial stem cells (BESCs), and normal bladder epithelial cells. It was found that BCSCs showed clonal homogeneity, and phylogenetic analysis indicated that BCSCs were derived from BESCs or BCNSCs. This study has confirmed that 21 abnormal key genes in BSCSs wherein 6 of which were not reported in bladder cancer in the past, which fully demonstrated the accuracy of single-cell sequencing (57). Faridani et al. demonstrated that microRNAs as the potential markers of different cell types and cell status by performing single-cell sequencing of the small-RNA transcriptome on human embryonic cells and tumor cells (58).

\section{Transcriptome Sequencing}

The transcriptome sequencing is a technique to sequence the transcript RNA, which is a sequence of cDNA that is reverse transcribed from mRNA, total RNA or other RNAs (e.g., small RNAs) to obtain the sum of RNA in a state of the cell. It was found to be sensitive and effective for intron fusion including oncogene activation caused by in-frame fusion. Kekeeva et al. performed high-throughput transcriptome sequencing on bladder cancer patients and screened 4 fusion introns in bladder cancer from 819 suspected fusion introns such as SEPT9/CYHR, IGF1R/TTC23, SYT8/TNNI2 and CASZ1/DFFA (59). Transcriptome sequencing can analyze gene expression profiles to reveal differentially expressed genes in tumors. Based on the transcriptome sequencing data, Zhang et al. confirmed the differential gene expressions of several bladder cancers through the comparison of bladder cancer and normal tissue transcriptome, in which ELF3 and MYBL2 are the tumor suppressors while $M E G 3, A P E X 1$, and $E Z H 2$ are the inducer of bladder cancer progression (60). In addition, transcriptome sequencing can also be applied to detect somatic mutations, but it is difficult to find a matching normal sample control which limits the application in this aspect. Liu et al. performed transcriptome sequencing of transitional cell carcinoma and identified 937 differentially expressed genes (61).

It is well known that miRNAs can form RNA silencinginducing complexes to inhibit translation of target mRNAs which is an important mechanism for gene expression regulation. Studies have shown that miRNAs also play an important role in tumor pathogenesis (62). Therefore, transcriptome sequencing technology has broad application prospects in detecting the abnormal miRNA expression in tumors.

\section{BLADDER CANCER GENOMICS AND PRECISION TREATMENT}

The traditional treatment strategy for bladder cancer is to formulate treatment plans according to grading and staging: early surgical treatment, middle and late stage surgery and perioperative radiotherapy and chemotherapy. However, the efficacy of treatment especially chemotherapy varies greatly in patients with the same type, grade, and stage of histology. It shows that there is still a considerable difference in the real situation of the patients with the same diagnosis. Since the differential sensitivity to chemotherapy in different patients, blind implementation of gold-standard chemotherapy is very likely to delay the treatment of chemotherapy-resistant patients. In response to the above problems, some researchers have proposed a strategy for precision medicine.

The essence of precise treatment of bladder cancer lies in the in-depth explanation of the molecular pathogenesis of bladder cancer. This is demonstrated on a more detailed molecular typing based on genome studies of bladder cancer. In addition, deletions of multiple tumor suppressor genes such as CDKN2A and $R B$, and amplification of oncogenes such as E2F3, SOX4, $E G F R$, and CCND1 were also identified. By further analyzing the mRNA and protein expression levels of the gene, bladder cancer is classified into 4 types. Type I and type II have the characteristics of breast cancer-like cells namely in which ERBB2 is highly expressed and estrogen receptor 2 (ESR2) pathway is activated. The difference is that type I mutations with fibroblast growth factor receptor 3 (FGFR3) and papillary histological 
phenotype III have similar characteristics of basal-like breast cancer cells, with the gene expression profile of squamous cells and stem cells, such as epidermal growth factor receptor (EGFR) and increased expression of keratin 5,6A and 14 and the like. Type IV is between type II and type III (32). This gene expression-based typing provides a scientific basis for the new molecular typing of bladder cancer. However, the relationship between molecular typing and clinical efficacy and prognosis still needs further exploration.

Genomics research can explore the genes involved in disease recurrence, prognosis, and drug sensitivity to effectively predict and evaluate the efficacy of chemotherapy for bladder cancer. Choi et al. had classified bladder cancer into three types by clustering the gene expression profiles of 183 cases of bladder cancer (6). Among them, luminal- and basal-like cells are same as the type I and type III of the TCGA type. The difference is that bladder cancer caused by luminal-like cells with wild type p53 and its activation of the signaling pathway is divided into a new category, known as the p53-like type. Combined with clinical prognosis and efficacy analysis, the luminal type has the best prognosis and is sensitive to neoadjuvant chemotherapy while basal type has the worst prognosis, about $60 \%$ of patients are not sensitive to neoadjuvant chemotherapy. The prognosis of p53-like type is between the two aforementioned, but almost insensitive to neoadjuvant chemotherapy. Choi's research provides a promising reference for the development of chemotherapy for bladder cancer (63). Roland et al. used a single-sample genomic subtyping classifier (GSC) to predict four consensus subtypes: luminal, luminal-infiltrated, basal, and claudin-low. They validated the clinical impact of these consensus subtypes in independent neoadjuvant chemotherapy (NAC) and non-NAC datasets. Luminal tumors showed the best overall survival (OS) with and without NAC. Luminal-infiltrated tumors were associated with poor prognosis with and without NAC. Compared with surgery alone, basal tumors had the most improved OS with NAC. Claudin-low tumors had poor OS regardless of treatment regimen (64). Despite the current lacking of well-defined predictive genetic markers in bladder cancer, above studies provide a clear research direction for precise treatment.

In addition, bladder cancer genomics can reveal the molecular mechanisms involved in the development and progression of bladder cancer and provide new molecular targets for the treatment of bladder cancer. To date, targeted therapy has achieved outstanding results in a variety of cancer treatments, such as gefitinib targeted therapy in treating lung cancer. However, bladder cancer targeted therapy is still at the early stage of clinical research, thus targeted therapy is not the option in guideline treatment recommendations for bladder cancer. At the present, some high-frequency abnormalities of bladder cancer that have been discovered and identified by genomics may become potential therapeutic targets. This includes signaling pathways such as EGFR, P13K/mTOR, HER-2 and FGFR3. Notably, the Food and Drug Administration recently approved erdafitinib as a treatment for patients with advanced urothelial cancer (UC) with FGFR3/2 mutations, who progressed on platinum-based chemotherapy. Clinical trials showed the overall response rate of erdafitinib was
$49 \%$ in patients with FGFR3 mutations $(65,66)$. Secondly, it is immunotherapy such as PD-1, and CTLA4. PD-1/PD-L1 immunotherapy can enhance the body's immune response to kill tumors and is effective for a variety of tumors including bladder cancer, lung cancer, stomach cancer, and kidney cancer. The US Food and Drug Administration (FDA) has approved this therapy for bladder cancer. Interestingly, bladder cancer has many tumor mutation burden (TMB). By analyzing the 443 bladder cancer samples from TCGA, single nucleotide polymorphism (SNP) and $\mathrm{C}>\mathrm{T}$ were the most frequent mutation types, and significant differences in tumor immune microenvironment were observed between the low TMB group and the high TMB group, including Mast cells resting, NK cells resting, T cells CD4 memory activated and $\mathrm{T}$ cells $\mathrm{CD} 8$, which makes it sensitive to immunotherapy (67). However, some patients is resistant to PD-1/PD-L1 immunotherapy, partially because TGF- $\beta$ in fibroblasts reduces anti-tumor immunity by inhibiting $\mathrm{CD} 8+\mathrm{T}$ cell infiltration into the tumor parenchyma (68).Fortunately, a personalized neoantigenbased vaccine, NEO-PV-01, was designed by whole exome and RNA sequencing of each patient's formalin-fixed tumor and matched normal cells from blood, and it consisted of high-quality neoepitopes encoded by somatic mutations and selected using bioinformatics algorithms. The clinical trial showed NEO-PV-01 in combination with PD-1 blockade was a safe and effective treatment to patients with bladder cancer by inducing $\mathrm{T}$ cells to traffic to the tumor and mediate cell killing (69). The third is cell cycle regulatory molecules, such as Aurora kinase A and Polo-like kinase I (3). The fourth is the antibody-drug conjugate (ADC), for example, enfortumab vedotin comprises an anti nectin-4 monoclonal antibody, protease cleavable linker and monomethyl auristatin E (MMAE). Because nectin-4 is highly expressed in all metastatic UC tumors, once the bonded enfortumab vedotin is internalized, the microtubule-disrupting agent MMAE is released leading to apoptosis of the tumor cell. Clinical trials demonstrated enfortumab vedotin is a new therapeutic method in patients with platinum- and immune checkpoint inhibitor-refractory disease $(70,71)$.

\section{CONCLUSION AND PROSPECT}

As shown Table 1, many genes are involved bladder cancer progression and metastasis, such as FGFR3, TP53, EGFR, HRAS and Ki67 (72-74), as well as HER2, TSC1, and ERCC1 which are the genes associated with bladder cancer. Among them, HER2 could be the potential therapeutic target, and ERCC1 is involved in the drug resistance of cisplatin and other chemotherapeutic drugs. Low expression of ERCC1 has a longer survival period. BRCA1 has DNA repair function and reduces the effect of chemotherapy drugs $(12,75,76)$. However, the current molecular markers that predict the prognosis and chemotherapy efficacy of bladder cancer patients are still at premature stages. Genetic markers that can accurately predict the therapeutic effect of bladder cancer is yet to found. In the future, intensive studies are needed to define the precisive molecular characterization of bladder cancer (77). Genomics is an effective tool for studying the molecular 
TABLE 1 | Common gene alterations in bladder cancer.

\begin{tabular}{|c|c|c|}
\hline Gene Name & Function & Referenc \\
\hline HER2 & Higher expression of HER2 indicates more aggressive tumor invasiveness. & $(10)$ \\
\hline ERCC1 & $\begin{array}{l}\text { ERCC1 is involved in the drug resistance of cisplatin and other chemotherapeutic drugs. Low expression of ERCC1 has a longer } \\
\text { survival period. }\end{array}$ & (13) \\
\hline EGFR,ERBB2, ERBB3 & Overexpression of EGFR, ERBB2 or ERBB3 is associated with tumor grade, stage, and prognosis in bladder cancer. & (19) \\
\hline PIKЗCA & Its mutation occurs in $25 \%$ of non-muscle invasive bladder cancer, associated with bladder cancer development and progression. & $(20)$ \\
\hline p16 & The loss of tumor suppressor genes (p16) often indicates the recurrence of low-grade bladder cancer. & $(21)$ \\
\hline p53,Rb,PTEN & Inactivate $p 53$ and pRb induces the invasion of tumor and metastasis of bladder cancer. & $(24,25)$ \\
\hline TERT & $\begin{array}{l}\text { TERT is highly expressed in invasive and advanced bladder cancer patients as compared to the early and non-invasive bladder } \\
\text { cancer patients. }\end{array}$ & (26) \\
\hline STAG2, ESPL1, NIPBL & $\begin{array}{l}\text { STAG2, ESPL1 and NIPBL genes with frequent mutations in bladder cancer are involved in the sister chromatid cohesion and } \\
\text { segregation process. }\end{array}$ & (44) \\
\hline ERCC2 & $\begin{array}{l}\text { ERCC2 mutations indicate a better prognosis of chemotherapy for bladder cancer and a lower rate of recurrence and metastasis } \\
\text { within } 2 \text { years. }\end{array}$ & $(50-52)$ \\
\hline ELF3, MYBL2 & Tumor suppressors. & (60) \\
\hline MEG3, APEX1, EZH2 & Inducer of bladder cancer progression. & $(60)$ \\
\hline FGFR3 & $\begin{array}{l}\text { The luminal-papillary subtype is characterized by FGFR3 mutations, fusion with transforming acid coiled-coil containing protein } 3 \\
\text { (TACC3), or amplification. }\end{array}$ & (65) \\
\hline
\end{tabular}

pathology of bladder cancer. It is believed that with advances in sequencing technology, genomics may bring more help to the targeted therapy of bladder cancer (78).

\section{AUTHOR CONTRIBUTIONS}

JZ and CL designed and supervised the review. YL and LS searched literature and wrote the manuscript. XG and NM reviewed of the manuscript. All authors contributed to the article and approved the submitted version.

\section{REFERENCES}

1. Tran L, Xiao JF, Neeraj Agarwal N, Duex JE, Theodorescu D. Advances in Bladder Cancer Biology and Therapy. Nat Rev Cancer (2021) 21(2):104-21. doi: 10.1038/s41568-020-00313-1

2. Cao Y, Tian T, Li W, Xu H, Zhan C, Wu X, et al. Long non-Coding RNA in Bladder Cancer. Clinica Chimica Acta (2020) 503:113-21. doi: 10.1016/ j.cca.2020.01.008

3. Porten SP. Epigenetic Alterations in Bladder Cancer. Curr Urol Rep (2018) 19 (12):102. doi: 10.1007/s11934-018-0861-5

4. Knowles MA, Hurst CD. Molecular Biology of Bladder Cancer: New Insights Into Pathogenesis and Clinical Diversity. Nat Rev Cancer (2015) 15(1):25-41. doi: $10.1038 / \mathrm{nrc} 3817$

5. Cai Z, Zhang F, Chen W, Zhang J, Li H. miRNAs: A Promising Target in the Chemoresistance of Bladder Cancer. OncoTargets Ther (2019) 12:11805-16. doi: 10.2147/OTT.S231489

6. Al-Ahmadie H, Netto GJ. Updates on the Genomics of Bladder Cancer and Novel Molecular Taxonomy. Adv Anatomic Pathol (2020) 27(1):36-43. doi: 10.1097/PAP.0000000000000252

7. Stratton MR. Exploring the Genomes of Cancer Cells: Progress and Promise. Science (2011) 331(6024):1553-8. doi: 10.1126/science.1204040

8. Garraway LA, Lander ES. Lessons From the Cancer Genome. Cell (2013) 153 (1):17-37. doi: 10.1016/j.cell.2013.03.002

9. Pleasance ED, Cheetham RK, Stephens PJ, McBride DJ, Humphray SJ, Greenman CD, et al. A Comprehensive Catalogue of Somatic Mutations From a Human Cancer Genome. Nature (2010) 463(7278):191-6. doi: 10.1038 /nature 08658

10. Soria F, Moschini M, Haitel A, Wirth GJ, Gust KM, Briganti A, et al. The Effect of HER2 Status on Oncological Outcomes of Patients With Invasive

\section{FUNDING}

The authors disclosed receipt of the following financial support for the research, authorship, and/or publication of this article: This work was supported by the National Natural Science Foundation of China (81672956, 81972390, 82002566), Beijing Science and Technology Projects (Z181100003818003), BeijingTianjin-Hebei Basic Research Cooperation Special Project (19JCZDJC65800(Z)), China Children and Teenagers' Fund (Y1803001), and Key Clinical Projects of Peking University Third Hospital (BYSY2017001).

Bladder Cancer. Urologic Oncol (2016) 34(12):533.e531-533.e510. doi: 10.1016/j.urolonc.2016.07.006

11. Hayashi T, Seiler R, Oo HZ, Jäger W, Moskalev I, Awrey S, et al. Targeting HER2 With T-DM1, an Antibody Cytotoxic Drug Conjugate, is Effective in HER2 Over Expressing Bladder Cancer. J Urol (2015) 194(4):1120-31. doi: 10.1016/j.juro.2015.05.087

12. Tschui J, Vassella E, Bandi N, Baumgartner U, Genitsch V, Rotzer D, et al. Morphological and Molecular Characteristics of HER2 Amplified Urothelial Bladder Cancer. Virchows Archiv an Int J Pathol (2015) 466(6):703-10. doi: 10.1007/s00428-015-1729-4

13. Weinstein JN, Akbani R, Broom BM, Lerner SP, Creighton CJ, Kim J, et al. Comprehensive Molecular Characterization of Urothelial Bladder Carcinoma. Nature (2014) 507(7492):315-22. doi: 10.1038/nature12965

14. Robertson AG, Kim J, Al-Ahmadie H, Bellmunt J, Guo G, Cherniack AD, et al. Comprehensive Molecular Characterization of Muscle Invasive Bladder Cancer. Cell (2017) 171(3):540-556.e25. doi: 10.1016/j.cell.2017. 09.007

15. Xu ZC, Cai HZ, Li X, Xu WZ, Xu T, Yu B, et al. ERCC1 C118T Polymorphism has Predictive Value for Platinum-Based Chemotherapy in Patients With Late-Stage Bladder Cancer. Genet Mol Res GMR (2016) 15(2):1-9. doi: 10.4238/gmr.15027801

16. Lee SJ, Lim JH, Choi YH, Kim WJ, Moon SK. Interleukin-28A Triggers Wound Healing Migration of Bladder Cancer Cells Via NF-kappaB-mediated MMP-9 Expression Inducing the MAPK Pathway. Cell Signal (2012) 24 (9):1734-42. doi: 10.1016/j.cellsig.2012.04.013

17. Lin J, Wang J, Greisinger AJ, Grossman HB, Forman MR, Dinney CP, et al. Energy Balance, the PI3K-AKT-mTOR Pathway Genes, and the Risk of Bladder Cancer. Cancer Prev Res (2010) 3(4):505-17. doi: 10.1158/19406207.CAPR-09-0263 
18. Shin K, Lim A, Zhao C, Sahoo D, Pan Y, Spiekerkoetter E, et al. Hedgehog Signaling Restrains Bladder Cancer Progression by Eliciting Stromal Production of Urothelial Differentiation Factors. Cancer Cell (2014) 26 (4):521-33. doi: 10.1016/j.ccell.2014.09.001

19. Forster JA, Paul AB, Harnden P, Knowles MA. Expression of NRG1 and its Receptors in Human Bladder Cancer. Br J Cancer (2011) 104(7):1135-43. doi: 10.1038/bjc.2011.39

20. Sjodahl G, Lauss M, Gudjonsson S, Liedberg F, Halldén C, Chebil G, et al. A Systematic Study of Gene Mutations in Urothelial Carcinoma; Inactivating Mutations in TSC2 and PIK3R1. PloS One (2011) 6(4):e18583. doi: 10.1371/ journal.pone. 0018583

21. Bartoletti R, Cai T, Nesi G, Nesi G, Girardi LR, Baroni G, et al. Loss of P16 Expression and Chromosome 9p21 LOH in Predicting Outcome of Patients Affected by Superficial Bladder Cancer. J Surg Res (2007) 143(2):422-7. doi: 10.1016/j.jss.2007.01.012

22. Kruger S, Mess F, Bohle A, Feller AC. Numerical Aberrations of Chromosome 17 and the 9p21 Locus are Independent Predictors of Tumor Recurrence in non-Invasive Transitional Cell Carcinoma of the Urinary Bladder. Int J Oncol (2003) 23(1):41-8. doi: 10.3892/ijo.23.1.41

23. Almendro V, Marusyk A, Polyak K. Cellular Heterogeneity and Molecular Evolution in Cancer. Annu Rev Pathol (2013) 8:277-302. doi: 10.1146/ annurev-pathol-020712-163923

24. Cordon-Cardo C. Molecular Alterations Associated With Bladder Cancer Initiation and Progression. Scand J Urol Nephrol Supplementum (2008) 218):154-65. doi: 10.1080/03008880802291915

25. Zhang ZT, Pak J, Huang HY, Shapiro E, Sun TT, Pellicer A, et al. Role of Haras Activation in Superficial Papillary Pathway of Urothelial Tumor Formation. Oncogene (2001) 20(16):1973-80. doi: 10.1038/sj.onc.1204315

26. Wu S, Huang P, Li C, Huang Y, Li X, Wang Y, et al. Telomerase Reverse Transcriptase Gene Promoter Mutations Help Discern the Origin of Urogenital Tumors: A Genomic and Molecular Study. Eur Urol (2014) 65 (2):274-7. doi: 10.1016/j.eururo.2013.10.038

27. Li C, Wu S, Wang H, Bi X, Yang Z, Du Y, et al. The C228T Mutation of TERT Promoter Frequently Occurs in Bladder Cancer Stem Cells and Contributes to Tumorigenesis of Bladder Cancer. Oncotarget (2015) 6(23):19542-51. doi: 10.18632 /oncotarget.4295

28. Marusyk A, Polyak K. Tumor Heterogeneity: Causes and Consequences. Biochim Biophys Acta (2010) 1805(1):105-17. doi: 10.1016/j.bbcan.2009.11.002

29. Pietras A. Cancer Stem Cells in Tumor Heterogeneity. Adv Cancer Res (2011) 112:255-81. doi: 10.1016/B978-0-12-387688-1.00009-0

30. Kreso A, O'brien CA, Galen VP, Gan OI, Notta F, Brown AMK, et al. Variable Clonal Repopulation Dynamics Influence Chemotherapy Response in Colorectal Cancer. Science (2013) 339(6119):543-8. doi: 10.1126/science.1227670

31. Lingner J, Hughes TR, Shevchenko A, Mann M, Lundblad V, Cech TR, et al. Reverse Transcriptase Motifs in the Catalytic Subunit of Telomerase. Science (1997) 276(5312):561-7. doi: 10.1126/science.276.5312.561

32. Lin SY, Elledge SJ. Multiple Tumor Suppressor Pathways Negatively Regulate Telomerase. Cell (2003) 113(7):881-9. doi: 10.1016/S0092-8674(03)00430-6

33. Artandi SE, Depinho RA. Telomeres and Telomerase in Cancer. Carcinogenesis (2010) 31(1):9-18. doi: 10.1093/carcin/bgp268

34. Huang FW, Hodis E, Xu MJ, Kryukov GV, Chin L, Garraway LA. Highly Recurrent TERT Promoter Mutations in Human Melanoma. Science (2013) 339(6122):957-9. doi: 10.1126/science.1229259

35. Killela PJ, Reitman ZJ, Jiao Y, Bettegowda C, Agrawal N, Diaz LAJr, et al. TERT Promoter Mutations Occur Frequently in Gliomas and a Subset of Tumors Derived From Cells With Low Rates of Self-Renewal. Proc Natl Acad Sci USA (2013) 110(15):6021-6. doi: 10.1073/pnas.1303607110

36. Borah S, Xi L, Zaug AJ, Powell NM, Dancik GM, Cohen SB, et al. Cancer. TERT Promoter Mutations and Telomerase Reactivation in Urothelial Cancer. Science (2015) 347(6225):1006-10. doi: 10.1126/science.1260200

37. Veronese L, Tournilhac O, Callanan M, Prie N, Kwiatkowski F, Combes P, et al. Telomeres and Chromosomal Instability in Chronic Lymphocytic Leukemia. Leukemia (2013) 27(2):490-3. doi: 10.1038/leu.2012.194

38. Ho L, Crabtree GR. Chromatin Remodelling During Development. Nature (2010) 463(7280):474-84. doi: 10.1038/nature08911

39. Chi P, Allis CD, Wang GG. Covalent Histone Modifications-Miswritten, Misinterpreted and Mis-Erased in Human Cancers. Nat Rev Cancer (2010) 10 (7):457-69. doi: 10.1038/nrc2876
40. Gui Y, Guo G, Huang Y, Hu X, Tang A, Gao S, et al. Frequent Mutations of Chromatin Remodeling Genes in Transitional Cell Carcinoma of the Bladder. Nat Genet (2011) 43(9):875-8. doi: 10.1038/ng.907

41. Wiegand KC, Shah SP, Al-Agha OM, Zhao Y, Tse K, Zeng T, et al. ARID1A Mutations in Endometriosis-Associated Ovarian Carcinomas. New Engl J Med (2010) 363(16):1532-43. doi: 10.1056/NEJMoa1008433

42. Dalgliesh GL, Furge K, Greenman C, Chen L, Bignell G, Butler A, et al. Systematic Sequencing of Renal Carcinoma Reveals Inactivation of Histone Modifying Genes. Nature (2010) 463(7279):360-3. doi: 10.1038/nature08672

43. Solomon DA, Kim T, Diaz-Martinez LA, Fair J, Elkahloun AG, Harris BT, et al. Mutational Inactivation of STAG2 Causes Aneuploidy in Human Cancer. Science (2011) 333(6045):1039-43. doi: 10.1126/science.1203619

44. Guo G, Sun X, Chen C, Wu S, Huang P, Li Z, et al. Whole-Genome and Whole-Exome Sequencing of Bladder Cancer Identifies Frequent Alterations in Genes Involved in Sister Chromatid Cohesion and Segregation. Nat Genet (2013) 45(12):1459-63. doi: 10.1038/ng.2798

45. Lawrence MS, Stojanov P, Polak P, Kryukov GV, Cibulskis K, Sivachenko A, et al. Mutational Heterogeneity in Cancer and the Search for New CancerAssociated Genes. Nature (2013) 499(7457):214-8. doi: 10.1038/nature12213

46. Margulies M, Egholm M, Altman WE, Attiya S, Bader JS, Bemben LA, et al. Genome Sequencing in Microfabricated High-Density Picolitre Reactors. Nature (2005) 437(7057):376-80. doi: 10.1038/nature03959

47. Schuster SC. Next-Generation Sequencing Transforms Today's Biology. Nat Methods (2008) 5(1):16-8. doi: 10.1038/nmeth1156

48. Meyerson M, Gabriel S, Getz G. Advances in Understanding Cancer Genomes Through Second-Generation Sequencing. Nat Rev Genet (2010) 11(10):68596. doi: $10.1038 / \mathrm{nrg} 2841$

49. Weir B, Zhao X, Meyerson M. Somatic Alterations in the Human Cancer Genome. Cancer Cell (2004) 6(5):433-8. doi: 10.1016/j.ccr.2004.11.004

50. Van Allen EM, Mouw KW, Kim P, Iyer G, Wagle N, Al-Ahmadie H, et al. Somatic ERCC2 Mutations Correlate With Cisplatin Sensitivity in MuscleInvasive Urothelial Carcinoma. Cancer Discovery (2014) 4(10):1140-53. doi: 10.1158/2159-8290.CD-14-0623

51. David L, Plimack ER, Censits JH, Garraway LA, Bellmunt J, Allen EV, et al. Clinical Validation of Chemotherapy Response Biomarker ERCC2 in Muscleinvasive Urothelial Bladder Carcinoma. JAMA Oncol (2016) 2(8):1094-6. doi: 10.1001/jamaoncol.2016.1056

52. Desai NB, Scott SN, Zabor EC, Cha EK, Hreiki J, Sfakianos JP, et al. Genomic Characterization of Response to Chemoradiation in Urothelial Bladder Cancer. Cancer (2016) 122(23):3715-23. doi: 10.1002/cncr.30219

53. Longo T, Mcginley KF, Freedman JA, Etienne W, Wu Y, Sibley A, et al. Targeted Exome Sequencing of the Cancer Genome in Patients With Very High-Risk Bladder Cancer. Eur Urol (2016) 70(5):714-7. doi: 10.1016/j.eururo.2016.07.049

54. Navin N, Krasnitz A, Rodgers L, Cook K, Meth J, Kendall J, et al. Inferring Tumor Progression From Genomic Heterogeneity. Genome Res (2010) 20 (1):68-80. doi: 10.1101/gr.099622.109

55. The biology of genomes. Single-Cell Sequencing Tackles Basic and Biomedical Questions. Science (2012) 336(6084):976-7. doi: 10.1126/science.336.6084.976

56. Navin N, Kendall J, Troge J, Andrews P, Rodgers L, McIndoo J, et al. Tumour Evolution Inferred by Single-Cell Sequencing. Nature (2011) 472(7341):90-4. doi: 10.1038/nature09807

57. Yang Z, Li C, Liu H, Liu H, Zhang X, Cai Z, et al. Single-Cell Sequencing Reveals Variants in ARID1A, GPRC5A and MLL2 Driving Self-renewal of Human Bladder Cancer Stem Cells. Eur Urol (2017) 71(1):8-12. doi: 10.1016/ j.eururo.2016.06.025

58. Faridani OR, Abdullayev I, Hagemann-Jensen M, Schell JP, Lanner F, Sandberg R. Single-Cell Sequencing of the Small-RNA Transcriptome. Nat Biotechnol (2016) 34(12):1264-6. doi: 10.1038/nbt.3701

59. Kekeeva T, Tanas A, Kanygina A, Alexeev D, Shikeeva A, Zavalishina L, et al. Novel Fusion Transcripts in Bladder Cancer Identified by RNA-Seq. Cancer Lett (2016) 374(2):224-8. doi: 10.1016/j.canlet.2016.02.010

60. Zhang M, Li H, Zou D, Gao J. Ruguo Key Genes and Tumor Driving Factors Identification of Bladder Cancer Based on the RNA-seq Profile. OncoTargets Ther (2016) 9:2717-23. doi: 10.2147/OTT.S92529

61. Liu Y, Noon AP, Aguiar Cabeza E, Shen J, Kuk C, Ilczynski C, et al. NextGeneration RNA Sequencing of Archival Formalin-Fixed Paraffin-Embedded Urothelial Bladder Cancer. Eur Urol (2014) 66(6):982-6. doi: 10.1016/ j.eururo.2014.07.045 
62. Mendell JT, Olson EN. MicroRNAs in Stress Signaling and Human Disease. Cell (2012) 148(6):1172-87. doi: 10.1016/j.cell.2012.02.005

63. Kandoth C, Mclellan MD, Vandin F, Ye K, Niu B, Lu C, et al. Mutational Landscape and Significance Across 12 Major Cancer Types. Nature (2013) 502 (7471):333-9. doi: 10.1038/nature12634

64. Seiler R, Ashab HAD, Erho N, van Rhijn BWG, Winters B, Douglas J, et al. Impact of Molecular Subtypes in Muscle-invasive Bladder Cancer on Predicting Response and Survival After Neoadjuvant Chemotherapy. Eur Urol (2017) 72(4):544-54. doi: 10.1016/j.eururo.2017.03.030

65. Casadei C, Dizman N, Schepisi G, Cursano MC, Basso U, Santini D, et al. Targeted Therapies for Advanced Bladder Cancer: New Strategies With FGFR Inhibitors. Ther Adv Med Oncol (2019) 11:1-14. doi: 10.1177/ 1758835919890285

66. Montazeri K, Bellmunt J. Erdafitinib for the Treatment of Metastatic Bladder Cancer. Expert Rev Clin Pharmacol (2020) 13(1):1-6. doi: 10.1080/ 17512433.2020.1702025

67. Lv J, Zhu Y, Ji A, Zhang Q, Liao G. Mining TCGA Database for Tumor Mutation Burden and Their Clinical Significance in Bladder Cancer. Biosci Rep (2020) 40(4):1-12. doi: 10.1042/BSR20194337

68. Mariathasan S, Turley SJ, Nickles D, Castiglioni A, Yuen K, Wang Y, et al. TGF- $\beta$ Attenuates Tumour Response to PD-L1 Blockade by Contributing to Exclusion of T Cells. Nature (2018) 554(7693):544-8. doi: 10.1038/ nature25501

69. Ott PA, Lieskovan SH, Bartosz Chmielowski B, Govindan R, Naing A, Bhardwaj N, et al. A Phase Ib Trial of Personalized Neoantigen Therapy Plus anti-PD-1 in Patients With Advanced Melanoma,non-Small Cell Lung Cancer, or Bladde Cancer. Cell (2020) 183(2):347-62.e24. doi: 10.1016/j.cell.2020.08.053

70. Alt M, Stecca C, Tobin S, Jiang DM, Sridhar SS. Enfortumab Vedotin in Urothelial Cancer. Ther Adv Urol (2020) 12:1-10. doi: 10.1177/1756287220980192

71. Park I, Lee JL. Systemic Treatment for Advanced Urothelial Cancer: An Update on Recent Clinical Trials and Current Treatment Options. Korean J Internal Medcine (2020) 35(4):834-53. doi: 10.3904/kjim.2020.204

72. Kompier LC, Lurkin I, Van Der Aa MN, van Rhijn BWG, van der Kwast TH, Zwarthoff EC. FGFR3, HRAS, KRAS, NRAS and PIK3CA Mutations in Bladder Cancer and Their Potential as Biomarkers for Surveillance and Therapy. PloS One (2010) 5(11):e13821. doi: 10.1371/journal.pone.0013821

73. Lopez-Beltran A, Luque RJ, Alvarez-Kindelan J, Quintero A, Merlo F, Carrasco JC, et al. Prognostic Factors in Stage T1 Grade 3 Bladder Cancer
Survival: The Role of G1-S Modulators (p53, p21Waf1, P27kip1, Cyclin D1, and Cyclin D3) and Proliferation Index (Ki67-MIB1). Eur Urol (2004) 45 (5):606-12. doi: 10.1016/j.eururo.2003.11.011

74. Wang L, Feng C, Ding G, Ding Q, Zhou Z, Jiang H, et al. Ki67 and TP53 Expressions Predict Recurrence of non-Muscle-Invasive Bladder Cancer. Tumour Biol J Int Soc Oncodevelop Biol Med (2014) 35(4):2989-95. doi: 10.1007/s13277-013-1384-9

75. Klatte T, Seitz C, Rink M, Rouprêt M, Xylinas E, Karakiewicz P, et al. ERCC1 as a Prognostic and Predictive Biomarker for Urothelial Carcinoma of the Bladder Following Radical Cystectomy. J Urol (2015) 194(5):1456-62. doi: 10.1016/j.juro.2015.06.099

76. Font A, Taron M, Gago JL, Costa C, Sánchez JJ, Carrato C, et al. BRCA1 mRNA Expression and Outcome to Neoadjuvant Cisplatin-Based Chemotherapy in Bladder Cancer. Ann Oncol Off J Eur Soc Med Oncol (2011) 22(1):139-44. doi: 10.1093/annonc/mdq333

77. Iyer G, Al-Ahmadie H, Schultz N, Hanrahan AJ, Ostrovnaya I, Balar AV, et al. Prevalence and Co-Occurrence of Actionable Genomic Alterations in HighGrade Bladder Cancer. J Clin Oncol Off J Am Soc Clin Oncol (2013) 31 (25):3133-40. doi: 10.1200/JCO.2012.46.5740

78. Plimack ER, Dunbrack RL, Brennan TA, Andrake MD, Zhou Y, Serebriiskii IG, et al. Defects in DNA Repair Genes Predict Response to Neoadjuvant Cisplatin-Based Chemotherapy in Muscle-invasive Bladder Cancer. Eur Urol (2015) 68(6):959-67. doi: 10.1016/j.eururo.2015.07.009

Conflict of Interest: CL was employed by Beijing Zhongke Jianlan Biotechnology Co., Ltd.

The remaining authors declare that the research was conducted in the absence of any commercial or financial relationships that could be construed as a potential conflict of interest.

Copyright $\odot 2021 \mathrm{Li}$, Sun, Guo, Mo, Zhang and Li. This is an open-access article distributed under the terms of the Creative Commons Attribution License (CC BY). The use, distribution or reproduction in other forums is permitted, provided the original author(s) and the copyright owner(s) are credited and that the original publication in this journal is cited, in accordance with accepted academic practice. No use, distribution or reproduction is permitted which does not comply with these terms. 